\title{
Progress of the APEX Experiment for Creation of an Electron-Positron Pair Plasma
}

\author{
Uwe Hergenhahn $^{1,2}$, Juliane Horn-Stanja ${ }^{1, a)}$, Stefan Niss ${ }^{1,3}$, Thomas Sunn \\ Pedersen $^{1}$, Haruhiko Saitoh ${ }^{1,4}$, Eve V. Stenson ${ }^{1}$, Matthew R. Stoneking ${ }^{5}$, Marcel \\ Dickmann $^{3}$, Christoph Hugenschmidt ${ }^{3}$, Markus Singer ${ }^{3}$, James R. Danielson ${ }^{6}$ and \\ Clifford M. Surko ${ }^{6}$ \\ ${ }^{1}$ Max Planck Institute for Plasma Physics, Greifswald and Garching, Germany \\ ${ }^{2}$ Leibniz Institute of Surface Modification, Permoserstr. 15, 04318 Leipzig, Germany \\ ${ }^{3}$ FRM II and Physics Department E21, Technische Universität München, 85747 Garching, Germany \\ ${ }^{4}$ The University of Tokyo, Kashiwa, Japan \\ ${ }^{5}$ Lawrence University, Appleton, WI, USA \\ ${ }^{6}$ Department of Physics, University of California, San Diego, La Jolla, CA 92093-0319, USA \\ ${ }^{a)}$ Corresponding author: juliane.stanja @ipp.mpg.de
}

\begin{abstract}
Electron-positron pair plasmas are an unexplored state of matter predicted to have properties intriguing for plasma physics as well as astrophysics. Here we described recent progress in the APEX collaboration dedicated to the production of a cold, confined electron-positron plasma in the laboratory. We focus on methods to inject positrons produced externally into a magnetic dipole trap, and to manipulate the ensuing trapped positron cloud. These experiments are carried out at the NEPOMUC positron beamline of the FRM II research reactor. Recent progress in producing more intense positron beams is briefly discussed.
\end{abstract}

\section{INTRODUCTION}

Experimenting with a confined electron-positron pair plasma is a vision that has fascinated plasma physicists for almost fourty years [1]. Since the first publication of a realistic plan to produce such plasmas [2], substantial progress has been made towards realization of this aim. In the APEX ( $A$ Positron-Electron plasma eXperiment) collaboration, we are working on a refined version of the aforementioned plan for pair plasma production (see [3]). Here we give an overview on our recent experimental results.

In contrast to many non-neutral, i.e. single-component plasmas, a pair plasma, having charge carriers of both sign, cannot be stored in a single linear particle trap. Nesting traps for species of opposite sign has been successful [4], but leaves the species in separate spatial regions of the traps or at different temperature. One configuration that has the promise of storing a plasma at any degree of neutrality is the magnetic dipole field of a current loop freely floating in space. Levitation of the current loop is vital to avoid particle losses on the supporting structure. In previous experiments, confinement of conventional (electron-ion) plasmas $[5,6]$ as well as pure electron plasmas [7] in the field of a levitated, superconducting ring conductor has been achieved.

Having a sufficiently intense source for positrons is another essential for the creation of an electron-positron plasma. The NEPOMUC positron beam line at the FRM II research reactor in Munich, Germany, will be used in our experiments [8]. Injecting the positron beam from NEPOMUC into our trapping geometry is a non-trivial task. In order to test injection strategies, we have constructed a mock-up device consisting of a permanent magnet that produces a dipole field and is surrounded by an electrode structure [9]. Important points to be addressed in this device include the following:

- achieving sufficient cross field transport to interface the NEPOMUC beam line to the trapping volume,

- manipulating the trapped positron cloud, e.g. to enable compression to a high-density state, and 
- understanding the trapping time and the underlying loss mechanisms.

In this contribution we will report about our progress with respect to these points. Accompanying the experiments, we have carried out extensive simulations of trajectories on the single particle level, which have greatly improved our insight into the injection mechanism. A general overview about earlier activities of the group has been given in [10].

Our group has also been active in characterizing the NEPOMUC positron beam with respect to, e.g., its intensity and energy components parallel and perpendicular to the magnetic guiding field [11]. Some results arrived at after publication of [11] are in the following section.

\section{PROPERTIES OF THE PRIMARY NEPOMUC BEAM}

The positron beam of NEPOMUC is produced from pair production in Pt foils, which at the same time act as positron moderator [8]. The actual beam energy is set by a bias potential applied to the Pt foil structure and the subsequent beam tube, which previously was chosen between 400-1000 V for most experiments. In order to increase the beam brightness, the ensuing 'primary' positron beam can be remoderated on a tungsten crystal in reflection geometry, which decreases its energy spread and spatial dimensions, however at the expense of a reduction in particle flux. Initial results reported by our group on injection of positrons into a dipole field also used this 'remoderated' beam [9].

As absolute number of (low-energy) positrons is the most decisive figure for our intended experiment, we have worked on transporting a low-energy primary beam to the open beam port of the NEPOMUC user platform. For easy diagnosis of the beam intensity and profile, we have mounted a retractable microchannel plate (MCP) detector in combination with a fluorescence screen upstream of our injection experiment. (This detector is now at the position of retractable metal plates, 'x-y target', used in our earlier work [11] to measure spatial beam extension.) As an alternative to the MCP, a copper electrode for measuring the absolute positron current can be connected to a charge-integrating amplifier (operational amplier AD820 with a 4.7-nF feedback capacitor).
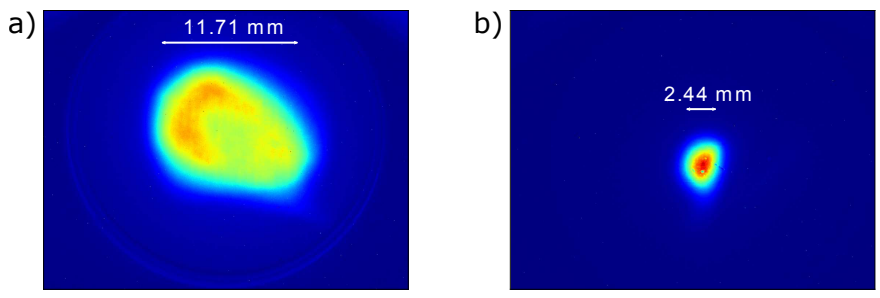

FIGURE 1. Color-coded intensity plot of (a) the $20-\mathrm{eV}$ primary and (b) the 5-eV remoderated positron beams at the open beam port. Intensity was recorded with an MCP detector read out via a fluorescence screen. The intensity scaling of the panels relative to each other is arbitrary.

A comparison of the spatial extent of the $20-\mathrm{eV}$ primary beam to the $5-\mathrm{eV}$ remoderated beam is shown in Figure 1 . As expected, the $20-\mathrm{eV}$ primary beam is more extended than its remoderated counterpart. Its size is similar to that measured for higher-energy primary beams at the so-called beam monitor 1, located farther upstream in the beam line [12], and at the open beam port [11]. The spatial profile found for the remoderated beam also is comparable with the linear dimensions found earlier [11].

TABLE 1. Compilation of some published intensity data for the primary and remoderated positron beams under various conditions.

\begin{tabular}{lccc}
\hline Location & $E_{k i n}(\mathrm{eV})$ & Primary & Remoderated \\
\hline Open beam port [11] & 22 & & $6.5(6) \cdot 10^{7}$ \\
Open beam port [11] & 400 & $5.4(1) \cdot 10^{8}$ & \\
Beam monitor 1 [12] & 540 & $1.14(7) \cdot 10^{9}$ & \\
\hline
\end{tabular}

The flux of the $20-\mathrm{eV}$ primary beam was determined to be about twice as high as that from the remoderated beam at comparable energy, given in Table 1. The difference is substantial, but less dramatic than expected. A possible reason is that most optimization efforts so far have been devoted to the $20-\mathrm{eV}$ remoderated beam, which was used in numerous 
other positron experiments. Compared to positron flux measured for higher-energy primary beams at various locations (Table 1) we find a reduction of up to $89 \%$. This seems to indicate that losses occur both because of the lower energy, possibly resulting in less efficient take-off of positrons from the Pt primary moderator into the beam line, as well as by non-adiabatic transport to the open beam port via three beam switches.

\section{POSITRON INJECTION INTO A MAGNETIC DIPOLE FIELD}

\section{Experiment Geometry}

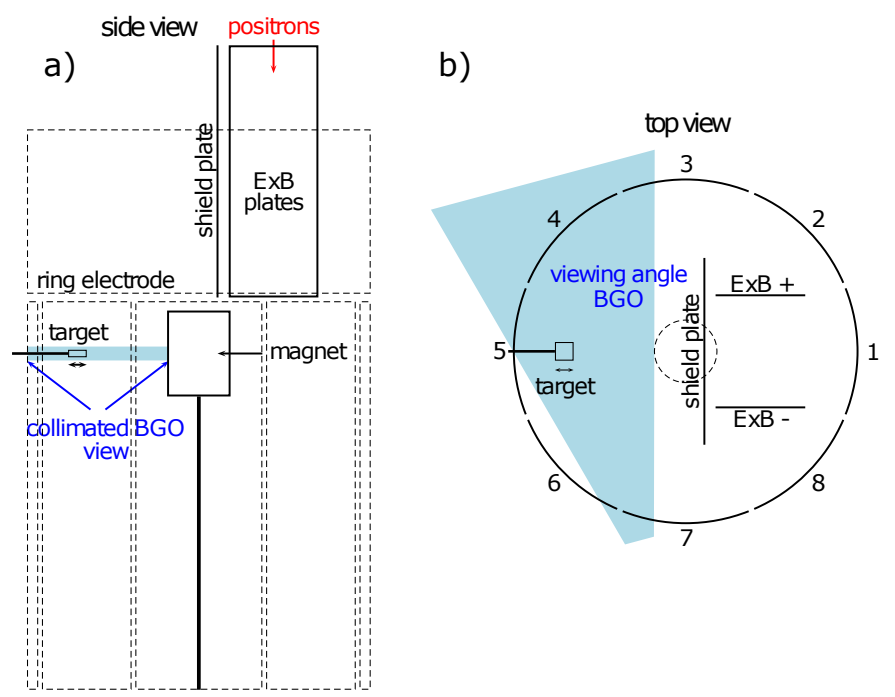

FIGURE 2. Sketch of the permanent magnet (center) and electrode arrangement to test injection of the NEPOMUC positron beam into a dipole field and manipulation of the injected positron cloud: (a) sideways view and (b) seen from top. Note the displacement between the axis of the NEPOMUC open beam port (pointing vertically downward, red arrow) and the central axis of the magnet. Intersecting the toroidal precession of positrons around the magnet, a target probe (horizontally oriented, grounded stainless steel plate, $1 \times 1 \mathrm{~cm}$ ) can be inserted via a feedthrough on the opposite side of the nominal point of injection. Positron annihilation on the target probe is monitored by a scintillation detector (BGO, see panel b). In order to increase specificity of the signal, its viewing angle is collimated by lead bricks to the shaded region. In earlier versions of this arrangement, electrode segments $1-8$ were pairwise connected $(1-2,3-4, \ldots)$ and extended up to the area now taken up by the separate, ring-shaped top electrode ('configuration A'), or were all at the same potential ('configuration 0') [9]. The configuration shown here will be referred to as 'B'.

A report about our first experiments with injecting positrons into the field of a magnetic dipole has been given [9]. Here we describe more recent results obtained after that publication. The geometry of our set-up, previously detailed in [9], is shown in Figure 2. It consists of a cylindrical permanent magnet with a field strength of $0.6 \mathrm{~T}$ at the pole faces, enclosed in a $\mathrm{Cu}$ housing. This magnet produces a dipole field with a vertical axis. The housing and the magnet support (shown in Figure $2 \mathrm{a}$ ) can be set to a bias voltage $U_{m a g}$. Slightly displaced from the central axis of the magnet, the NEPOMUC positron beam enters from above, passing between a pair of rectangular electrodes. Typically, as indicated in Figure $2 \mathrm{~b}$, the plates are set to voltages $U_{E \times B},-U_{E \times B}$ of equal absolute value but opposite sign, in order to avoid a net electrostatic acceleration or deceleration of the beam. The directions of the electric and magnetic fields between the plates are such that an $E \times B$-drift transports the positrons across magnetic field lines. For suitably chosen potentials, some positrons are able to enter a region of the magnetic dipole field in which they are trapped by magnetic mirroring when moving towards the poles of the magnet. This leads to an up-down bounce movement of the positrons, to which a much slower precession in the equatorial direction around the magnet is added [9].

In order to manipulate the positron cloud precessing around the magnet, a cylindrical electrode set (bias voltages $\left.U_{\text {seg } 1-8}\right)$ has been mounted along the edge of the vacuum vessel. Over the course of our experiments its arrangement has evolved; the most recent configuration is shown in Figure 2, and for the sake of discussion will be referred to as 'B'. Two simpler arrangements of the electrodes are not explicitly shown, but points in which they differ from 
the Figure are explained in the caption. Those will be referred to as ' 0 ' and ' $\mathrm{A}$ '. For the simplest configuration ' 0 ', injection of positrons into the dipole field with an efficiency of $40 \%$ was shown in [9], but understanding of the underlying mechanism was incomplete, and an optimization strategy towards higher efficiencies remained unclear.

\section{Particle Trajectory Simulations}

In order to make progress on these problems, we have carried out simulations of numerous injection conditions, and compared them to the experimental outcomes. A very satisfactory agreement has been reached, as will be demonstrated by some examples.

In the current study, the density of the positron beam is sufficiently low to allow a treatment on a single particle basis. This can be justified by an estimate of the positron density in the particle trap. As discussed in detail below, only a short bunch of particles from the Nepomuc beam is present in our trap at any time. Putting in some numbers $\left(3 \times 10^{7} e^{+} / \mathrm{s}, 100 \mu\right.$ s bunch positron bunch length, trapping volume estimated by a hollow cylinder with $h=50 \mathrm{~mm}$, $r_{i}=30 \mathrm{~mm}, r_{o}=70 \mathrm{~mm}$ ) leads to particle densities on the order of $5 \mathrm{~cm}^{-3}$. The Debye length associated with this plasma would be on the order of $3.6 \mathrm{~m}$, which is much larger than its linear dimension. This is another way of saying that kinetic effects are much more important than self-generated electric fields from the plasma (see [13]). The problem of simulating the positron trajectories therefore boils down to propagating a particle under the influence of a static electromagnetic field. We have used the commercial software SIMION 8.1.1.32 [14] for this purpose. In the following, we describe technical details of our simulations.

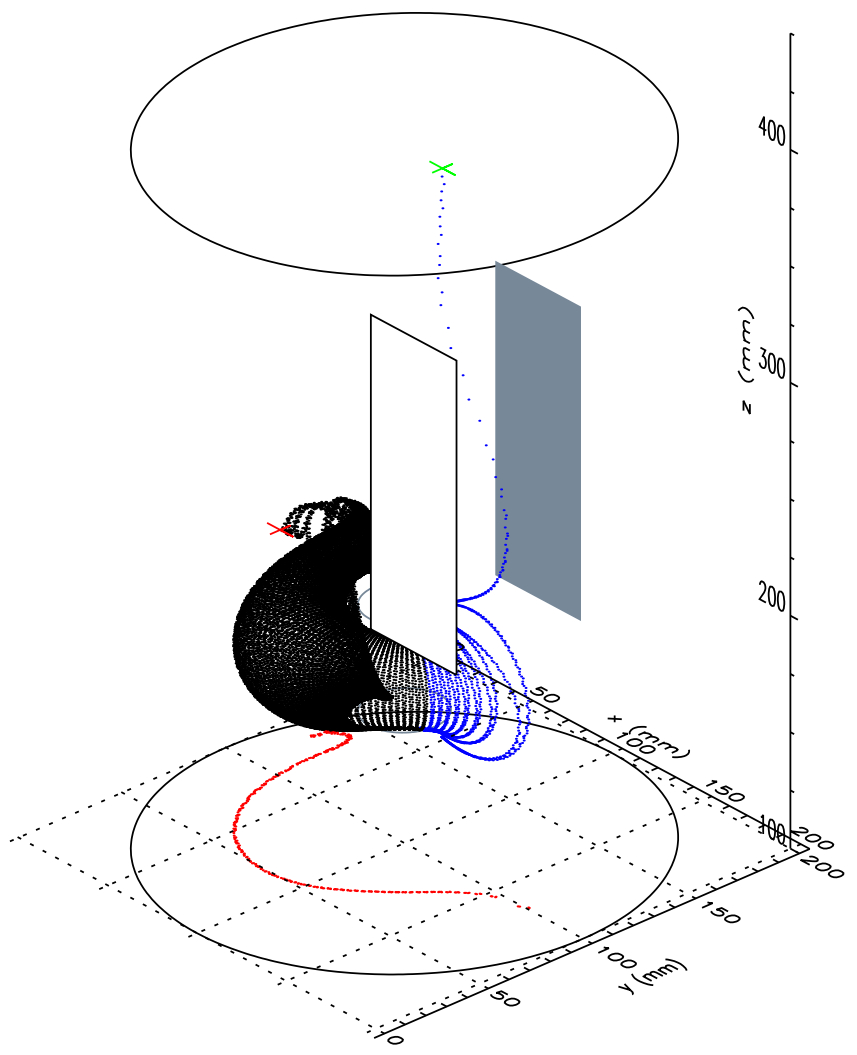

FIGURE 3. Typical simulated single particle trajectory, conditions: positron beam with $\left\langle E_{\|}\right\rangle=5.16 \mathrm{eV},\left\langle E_{\perp}\right\rangle=0.78 \mathrm{eV}$; electrode voltages $\mathrm{U}_{E \times B}= \pm 175 \mathrm{~V}, \mathrm{U}_{\text {mag }}=-15 \mathrm{~V}, \mathrm{U}_{\text {seg } 1-8}=+5.5 \mathrm{~V}$. The particle trajectory during the first $1 \mu$ s of simulated flight time is drawn in blue. The red trace at the bottom is a projection of the points where the particle trajectory intersects the equatorial plane. Contours are drawn to mark the edges of the vacuum vessel, the magnet and the $E \times B$-plates.

Due to the lack of symmetry of the set-up, a fully three-dimensional geometry had to be simulated. To keep the requirements on core memory manageable, a grid with a mesh size of $1 \mathrm{~mm}$ was used. Particles were propagated by a fourth order Runge-Kutta scheme. The step size was set to 0.05 grid units, and could be further reduced under the 
control of SIMION in regions where strong acceleration occurred (T.Qual = 119). Sufficient numerical accuracy with these parameters was tested by simulating precession in a pure dipole field over $50 \mu$ s. Since SIMION is doing a straightforward calculation of the particle trajectories, it is numerically ineffective in regions with a strong magnetic field, in particular near to the permanent magnet. This is because a large number of tiny iteration steps is needed to simulate the cyclotron motion around the magnetic field lines, which has a gyroradius of typically $10-100 \mu \mathrm{m}$. As the gyroradius is smaller than any other relevant length scale of the problem, in principle the guiding center approximation [15] could be used to make calculations more effective. We are not aware of a code that implements it in arbitrary geometries, though. Some improvements in numerical efficiency (not covered here) were achieved recently by changing from SIMION's Runge-Kutta scheme to an internally developed code using a symplectic integrator.

The simulation region encompassed the vacuum chamber housing the permanent magnet and the transfer electrodes. Its upper end is at the interface to a section of our apparatus used for beam diagnosis (see [9, 11]). The guiding field of the NEPOMUC positron beam line is connected to the dipole trap by a pair of Helmholtz coils. For our simulations, the magnetic field was calculated from the coil parameters using analytical expressions for the field of a thin current loop (see e.g. [16]). The permanent magnet was modelled by applying the same expressions to an assembly of current loops mimicking the spatial dimensions of the magnet and the field on its pole face.

A remoderated positron beam with a nominal energy of $5 \mathrm{eV}$ was simulated. For each simulation run, a starting position in the $z=400 \mathrm{~mm}$ plane was fixed. The positron beam in that position was represented by a set of, typically, 100 particles. Earlier, we found the remoderated beam is well characterized by independent distribution functions for the perpendicular and the parallel energy [11]. Following this work, for the perpendicular energy $E_{\perp}$ we have used a Maxwell-Boltzmann distribution according to

$$
f\left(E_{\perp}\right)=\frac{1}{k_{B} T_{\perp}} \exp \left(-\frac{E_{\perp}}{k_{B} T_{\perp}}\right),
$$

with a temperature parameter of $T_{\perp}=0.78 \mathrm{eV}$. For the parallel energy $E_{\|}$a Gaussian distribution was assumed,

$$
f\left(E_{\|}\right)=\frac{1}{\sqrt{2 \pi} \sigma_{\|}} \exp \left(-\frac{\left(E_{\|}-\left\langle E_{\|}\right\rangle\right)^{2}}{2 \sigma_{\|}^{2}}\right) \text {, }
$$

with a mean of $5.16 \mathrm{eV}$ and a spread $\sigma_{\|}$that was set equal to $T_{\perp}$. Initial energies for the particles were produced as random draws from these distribution functions. The parallel movement of the particle was aligned to the direction of the magnetic field at the starting point, and each particle was given a random gyration angle. The particles were propagated one at a time.

\section{Discussion of Injection Results}

A simulated trajectory is shown in Figure 3. Here, we have simulated the conditions chosen in our original work [9]. It can clearly be seen how particles transit from guided flight in the transport field to a trapped bounce motion around the magnet. For the sake of discussion, after $1 \mu$ s of simulated flight a particle is categorized as having been injected into the trapping field.

It is instructive to connect the particle motion to the topology of the magnetic field in our experiment. Towards this end, we have visualized the magnetic field lines as the iso-surfaces of the flux function $\Psi(\mathbf{r})$. The following expression for the flux function of a thin current loop (radius $a$, center at $\left(0, z_{c}\right)$ ), expressed in cylindrical coordinates, was used:

$$
\Psi(r, z):=2 \pi r A_{\phi}=\mu_{0} I \frac{2}{k} \sqrt{a r}\left[\left(1-\frac{k^{2}}{2}\right) K(k)-E(k)\right] .
$$

Here, $A_{\phi}$ is the vector potential, $I$ is the current, $K$ and $E$ are complete elliptical integrals of the first and second kind, and $k$ the abbreviation for $k(r, z)=\left[4 a r /\left((a+r)^{2}+\left(z-z_{c}\right)^{2}\right)\right]^{1 / 2}$.

In order to be trapped, the positrons have to transit from the system of open field lines originating at the source to the system of closed dipole field lines around the magnet. A separatrix marking the division between the two systems is plotted red in Figure 4, which shows that injection is accomplished by a combination of $E \times B$-drift induced crossfield transport and magnetic mirroring. Note that the lower end of the $E \times B$-electrodes is at $z=0.21 \mathrm{~m}$. We also note that the overlap of the dipole fields from Helmholtz coils and magnet lead to the occurrence of an X-point, which lies outside of the region we have chosen for cross-field transport, however. 


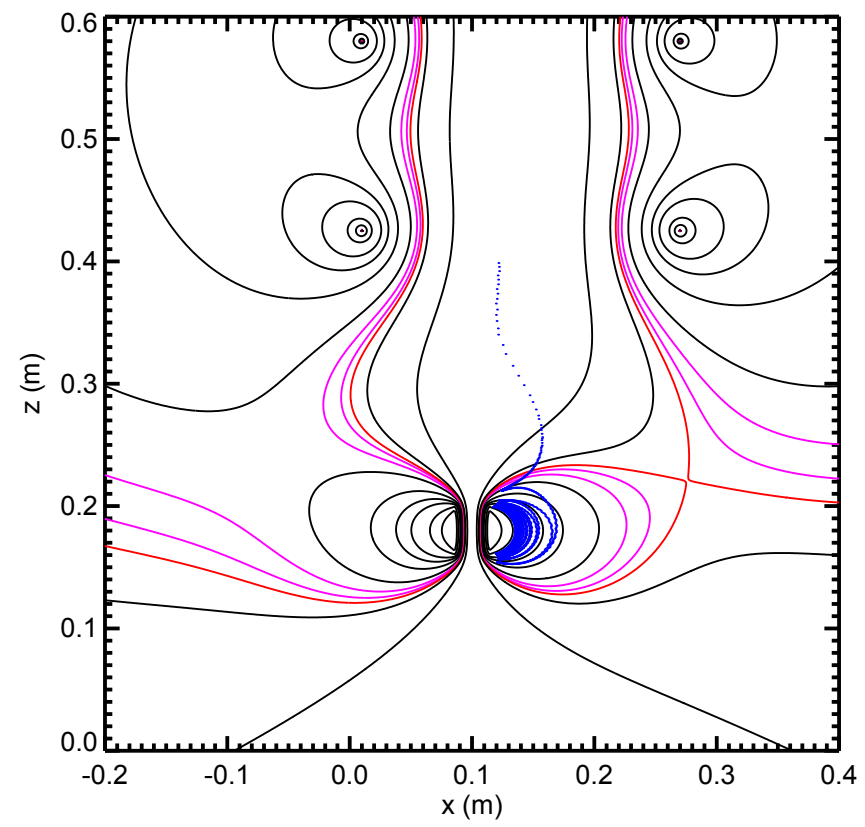

FIGURE 4. Flux surface contours in the plane containing the magnet dipole vector and the central axis of the NEPOMUC guiding field. The field of view contains the magnet at $x=0.1 \mathrm{~m}$ as well as Helmholtz coils for beam guiding. The blue trajectory shown in Figure 3 is projected onto this plane.

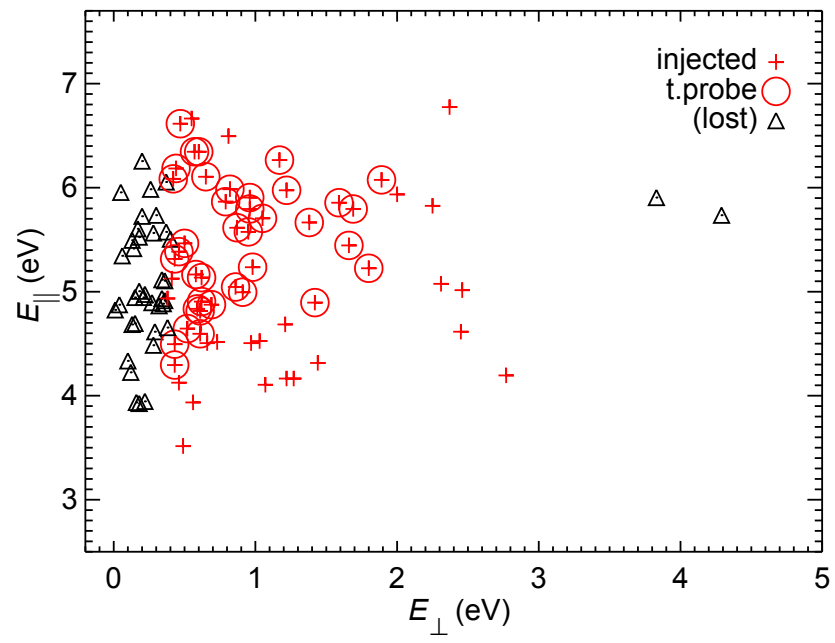

FIGURE 5. Injection as a function of the parallel and perpendicular energy of simulated trajectories, same conditions as in Figure 3. Particles injected into the dipole field are drawn by a '+'-symbol, particles that are not injected by a triangle. Particles that make at least a half turn around the magnet, thereby reaching the target probe, are additionally marked by an open circle.

The experimentally measured injection efficiency in [9] was found to be approx. $40 \%$. It was determined from the fraction of current measured on the target probe, compared to an electrode in the upstream section for beam analysis. The fraction of simulated particles that reach the target probe was determined by post-processing of the trajectories; electrostatic effects induced by insertion of the probe were thus neglected. For the ensemble shown in Figure 5 , a fraction of 0.38 reaches the target probe, in good agreement with the experiment. Inspecting the particle energies of the ensemble shows that injection mostly fails for particles with a low $E_{\perp}$ (Figure 5). Moreover, particles with insufficient $E_{\|}$may enter the dipole field region, but are not able to further precess around the magnet; in these particular injection conditions this is caused by the positive bias voltage on all wall electrodes. 


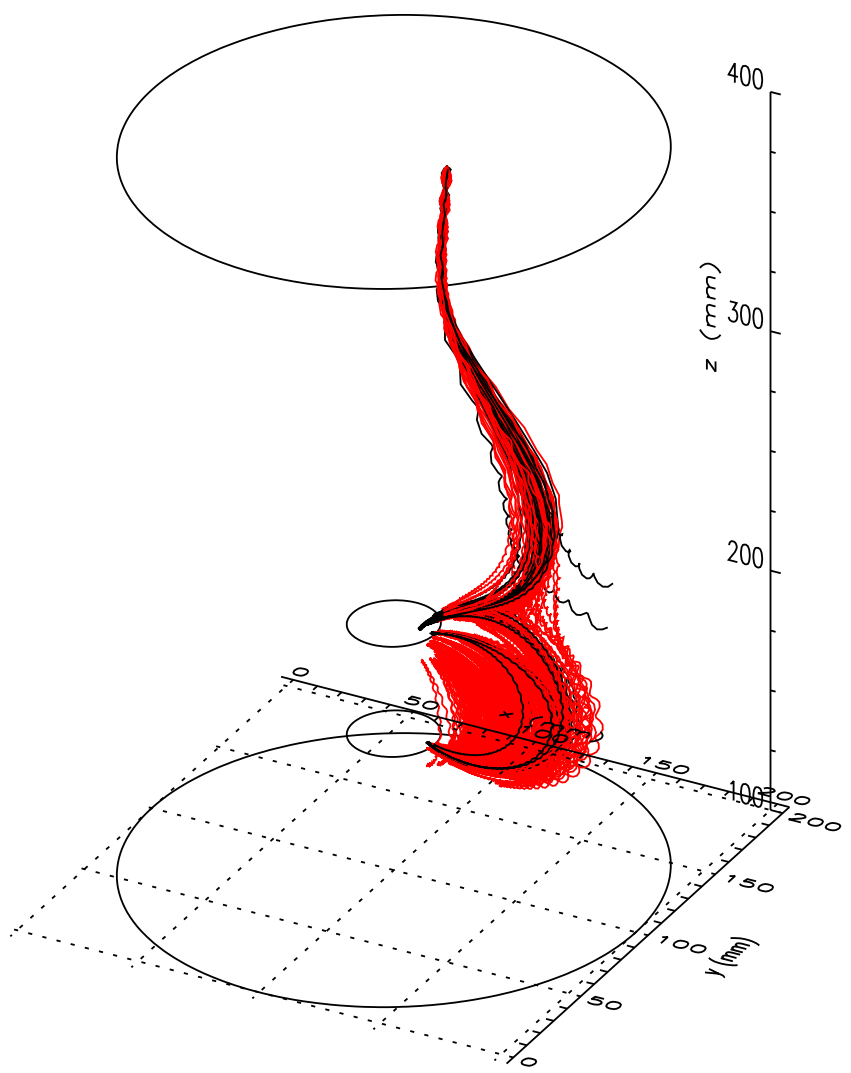

FIGURE 6. Simulated trajectories for an ensemble of onehundred particles with randomized starting conditions, same settings as in Figure 3. The first $\mu$ s of simulated flight time (or the time until a particle splats on an electrode), is shown. Trajectories of injected particles (total simulated flight time $>1 \mu \mathrm{s}$ ) are shown in red.

In order to further analyze particle losses in the injection process, we have plotted the trajectories of the whole simulated ensemble in Figure 6. It can be seen that most non-injected particles directly, or after one bounce, splat on the magnet. This points to the role of magnetic mirroring in the injection process: Because the magnetic fieldlines of the guiding field connect to the pole face of the magnet, a loss cone exists for particles with insufficient $E_{\perp}$. The presence of $E \times B$-drifts may only change the size of this loss cone (by moving particles to a field line that corresponds to different field strengths) but cannot prevent losses on the face of the magnet entirely. The effect of the inhomogenity of the fields is seen by the example of some particles which undergo one or two bounces when approaching the magnet, but then splat on its surface nevertheless. It is interesting to speculate whether such particles could be trapped in the next version of our device, which will use a levitated, superconducting current loop [17]. For the time being, the loss cone can be blocked or eliminated by other means, e.g. biasing the magnet positively.

Figure 7 shows first results using this approach. In electrode configuration A, positrons from the $5 \mathrm{eV}$ remoderated beam were injected, and annihilation counts on the target probe (after a $180^{\circ}$ precession around the magnet), minus a background signal with the probe retracted, were recorded. Counts were recorded for a series of $\mathrm{U}_{\text {mag }}$ bias voltages. Obviously, setting all segments and the magnet to a positive bias voltage creates a volume that positrons cannot enter electrostatically (diamonds). However, if this condition on the wall segments is released, a positive $\mathrm{U}_{m a g}$ indeed leads to more effective injection than neutral or negative values. It also leads to more effective injection than the original scheme of [9], which is similar to the all biased data set in Figure 7. We have achieved up to $100 \%$ injection efficiency by this approach, which will be detailed in a forthcoming publication [18].

Results discussed so far have been obtained for particles using a single starting point, while in fact the positron beam has an extension of at least a few $\mathrm{mm}$ (Figure 1). We have repeated our simulations for a number of starting points in the $z=400 \mathrm{~mm}$ plane, and have found that injection efficiency does not depend very sensitively on this variable. In other words, the spot giving best injection conditions has an extension which qualitatively matches the 


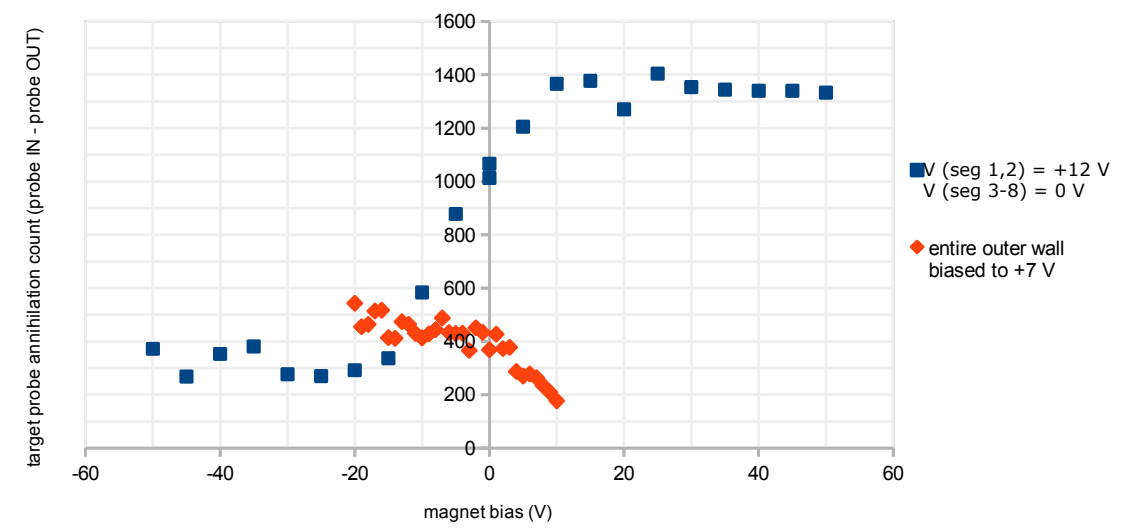

FIGURE 7. Counts on target probe for varying magnet bias. The wall electrodes either were biased all positively (diamonds), or only $25 \%$ of the wall, near the injection region, was biased (squares).

actual beam dimensions. (No causal relation between the two facts is known to us.)

After somewhat more than a half revolution around the magnet, the simulated trajectory shown in Figure 3 ends with a splat of the particle on one of the outer electrodes. (We note that the total energy of this selected particle is sufficient to reach the outer electrodes despite them being on a $+5.5 \mathrm{~V}$ bias potential.) Obviously, this inhibits particle trapping over extended times. We have extensively investigated the cause of this effect, which is universal to the particle ensemble and robust against variations of the electrode settings. It can be traced back to the electrostatic fringe field of the positively charged $E \times B$-electrode, which leaks into the part of the trapping region pertaining to approx. $270^{\circ}-330^{\circ}$ of revolution around the magnet. No easy fix has occurred to us yet, as lowering the absolute value of the $E \times B$-voltage leads to less efficient injection, and increasing the particle energy requires an increase of the $E \times B$-voltage as well. We therefore have conducted experiments on positron trapping times by injecting a bunch of particles, and subsequently switching out the $E \times B$-voltage, as developed empirically in [9]. Some results on particle trapping are described in a later section of this report.

\section{TRAPPED PARTICLE MANIPULATION}

Some control on the trapped particle orbit is possible in the injection process; our results on this aspect will be given elsewhere [18]. Here, we focus on methods to manipulate the positron cloud during precession around the magnet. For non-neutral plasmas stored in a Penning-Malmberg trap, compression of the plasma filament by AC fields applied to a radially segmented electrode is very effective [19]. We have attempted to implement an analogue to these 'rotating wall' (RW) fields in our set-up. To this end, sinusoidally varying voltages produced by a function generator were applied to the electrode segments shown in Figure 2. Segments were paired, as only four output channels were available at the time of experiments. Between each pair of electrodes, a phase lag of $\pi / 2$ was applied, so the field circulated around the magnet. We will therefore call it a RW field, although the mechanism of its action (if any) has to be different from RW fields in a Penning-Malmberg. While the latter injects torque into the rotating non-neutral plasma, the former instead induces an additional $E \times B$-drift that may drive particles inward. These experiments used an electrode configuration with the top ring set to $12 \mathrm{~V}$.

In order to find whether these AC fields produce any effect, we have monitored annihilation counts on the target probe (Figure 8) for continuous injection of positrons into the trap in the presence of a RW field with variable frequency. These experiments were done in configuration $\mathrm{B}$, using a magnet bias of $\mathrm{U}_{m a g}=-5 \mathrm{~V}$ and $\mathrm{U}_{E \times B}= \pm 230 \mathrm{~V}$. The amplitude of the field was limited by the function generator. Using an oscilloscope we measured $14 \mathrm{~V}$ for frequencies up to $1.5 \mathrm{MHz}$, but lower amplitudes at higher frequencies, caused by the frequency response of the amplifiers used. The idea was to insert the target probe to a position where it intercepts the positron cloud, thus producing some amount of annihilation counts. If the annihilation rate decreases by application of the RW field, this could indicate an inward drive of the particles, which now pass the probe without annihilating.

In order to extract experimental information on the RW field effects from Figure 8, for each probe position the data measured with the field on (symbols) should be compared with the respective zeroline (dashed). For the outermost probe position, a decrease is seen for all frequencies below $1 \mathrm{MHz}$. At more inward positions of the probe, 

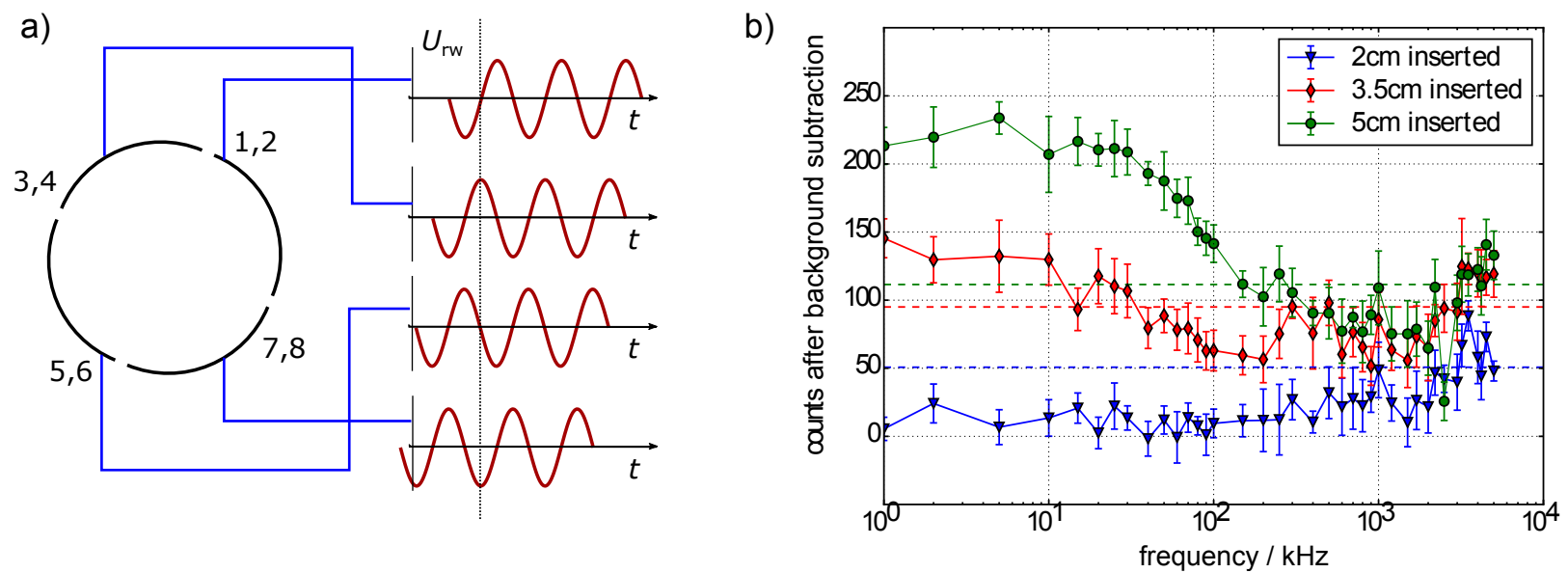

FIGURE 8. Effect of applying a rotating field to the electrodes around the trapping volume. a) Sketch of phase and waveform of the electric field. The oscillating field ('RW field') was applied continuously. Electrodes are seen from the top, and numbering of the segments refers to Figure 2. b) Annihilation counts on the target probe measured in the presence of a RW field. Data are shown vs. RW frequency for three different target positions, probing (from 2 to $5 \mathrm{~cm}$ insertion) gradually more central regions of the trapping volume. Insertion by $3.5 \mathrm{~cm}$ approximately corresponds to a 'halfway in' position; at $5 \mathrm{~cm}$ the full positron cloud should be blocked. A background signal measured with retracted probe has been subtracted. Dashed lines give the respective signal without a RW field. See text for details.

an increase or a decrease in counts can be seen, depending on the frequency. Inward compression is also suggested by the data measured at $3.5 \mathrm{~cm}$ for frequencies of one to several hundred $\mathrm{kHz}$. The conclusion from this Figure is that manipulation of the shape of the injected positron packets by a RW field is possible. For compression, the most suitable frequency range seems to be $150-200 \mathrm{kHz}$.

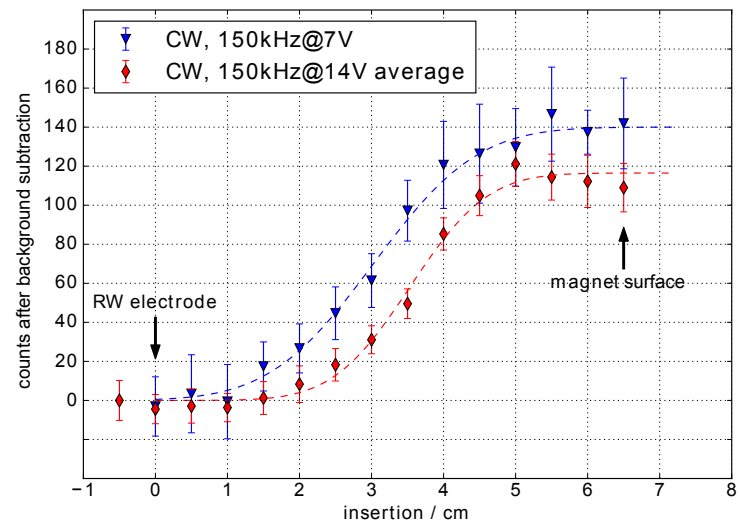

FIGURE 9. Annihilation counts on the target probe measured for two different amplitudes of a RW field, otherwise same conditions as in Figure 8.

For a RW field with $150 \mathrm{kHz}$, we have recorded an extended set of annihilation counts vs. probe position data. Results for two different RW amplitudes are in Figure 9. Differences between the two settings are clearly visible (showing again that the fields have an effect), higher amplitude fields lead to a more efficient inward drive.

At the same time, however, in the plateau region where the probe blocks the full injected positron beam, fewer positrons are observed for the higher field amplitude. We attribute this to an influence of the RW field on the injection process itself, which may be constructive or detrimental. A similar finding is suggested by inspecting the 5-cm trace in 
Figure 8; for values of the frequency below some tens of $\mathrm{kHz}$, we see an increase of positron counts despite Figure 9 telling us that the probe already intercepts the full cloud. For this reason, we have refined our strategy by going to a pulsed injection scheme, which allows a targeted use of short RW wave trains. These experiments are described in a separate contribution in this volume [20].

\section{TRAPPED ENSEMBLE LIFETIME}

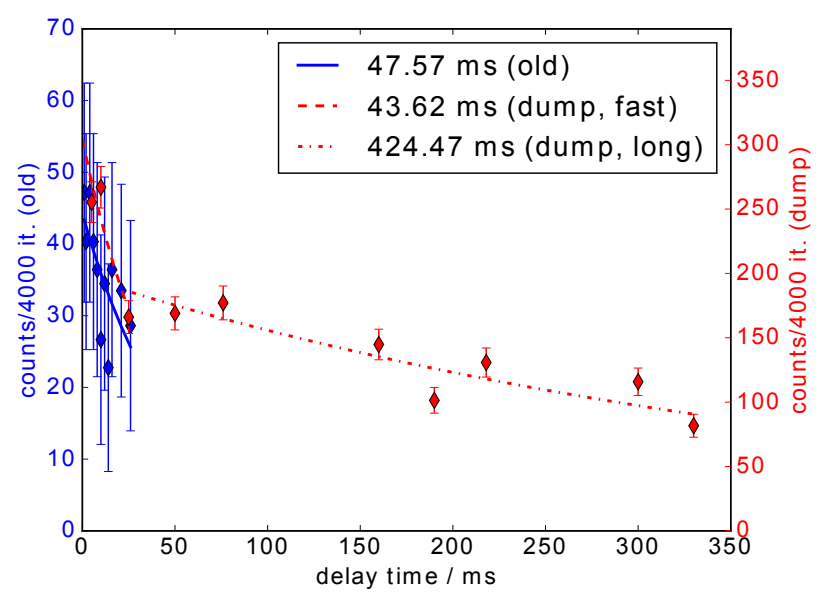

FIGURE 10. Lifetime of positron clouds trapped in our magnetic dipole field, measured by two different acquisition schemes (blue symbols, referred to lhs axis and red symbols, referred to rhs axis). Data indicate the presence of two components, one short-lived, the other being present for hundreds of ms. See text for details.

The lifetime of the injected positron cloud is an essential parameter for our experiments on pair plasmas, as the confinement of about $10^{10}$ positrons in our magnetic trap (together with the same amount of electrons) will be needed to plausibly fullfill the requirements for formation of an electron-positron plasma [3, 13]. (For the example of the 22$\mathrm{eV}$ remoderated beam with intensity given above this corresponds to $154 \mathrm{~s}$ of prefectly efficient injection.) We have continued experiments in which we measure the lifetime of a short bunch of injected positrons; initial results measured lifetimes of several ms (hundreds of toroidal precessions) [9]. In order to improve sensitivity of these measurements, we have changed the data acquisition scheme. In our first experiments, to measure a lifetime with an expected upper limit of $\tau$, a counting window with a temporal duration $t_{w}<<\tau$ was started after a delay time $\Delta t$ with respect to the start of the trapping phase; $\Delta t$ was then scanned. To acquire usable statistics, acquisition for each $\Delta t$ had to be iterated several thousand times. The shortcoming of this scheme, which was initially dictated by the electronics components available to us, is that only a fraction of each period $\tau$ is used for data acquisition. Instead of detecting the positrons lost during the confinement phase, we now dump the positron cloud after a delay time $\Delta t \leq \tau$ and record the amount of positrons left in the trap. This lead to a greatly improved signal-to-noise ratio for a given wall-clock time of positron beam availability.

In Figure 10, data acquired with the old and new schemes are compared. These experiments were done in configuration $\mathrm{B}$, using a magnet bias of $\mathrm{U}_{\text {mag }}=5 \mathrm{~V}, \mathrm{U}_{E \times B}= \pm 190 \mathrm{~V}, \mathrm{U}_{\text {top }}=16 \mathrm{~V}$ and $\mathrm{U}_{\text {seg } 1-8}=0 \mathrm{~V}$. The new acquisition scheme clearly revealed the presence of two fractions of positrons in the trapped particle cloud, one with a lifetimefor the electrode configuration probed here-of approx. $44 \mathrm{~ms}$, and another that is far more long-lived (lifetime about $424 \mathrm{~ms}$ ). Although these results are yet not sufficient to readily produce a pair plasma, we consider them extremely encouraging.

\section{SUMMARY AND OUTLOOK}

En route to the production of a cold, magnetically confined electron-positron plasma, we have systematically studied the injection of a positron beam from a reactor based source into a magnetic dipole geometry. A comparison of experiments with trajectory simulations lead us to a good understanding of the factors that are decisive for efficient 
injection. In a proof-of-principle experiment an inward drive of the injected positrons due to their interaction with AC fields has been observed. Trapping of positrons with lifetimes in the hundreds of ms has been demonstrated. As the next step in the evolution of this long-term project, we will now focus on magnetic trapping by the field of a levitated, superconducting current loop.

\section{ACKNOWLEDGMENTS}

This work is based upon experiments performed at the NEPOMUC instrument operated by FRM II at the Heinz Maier-Leibnitz Zentrum (MLZ), Garching, Germany. Funding by the Deutsche Forschungsgemeinschaft (Hu 978/15, Sa 2788/2) is gratefully acknowledged. UCSD would like to acknowledge support from the UCSD Foundation. EVS acknowledges a grant by the Helmholtz Postdoc-Programme.

\section{REFERENCES}

[1] V. Tsytovich and C. B. Wharton, Comments Plasma Phys. Control. Fusion 4, 91-100 (1978).

[2] T. Sunn Pedersen, J. R. Danielson, C. Hugenschmidt, G. Marx, X. Sarasola, F. Schauer, L. Schweikhard, C. M. Surko, and E. Winkler, New J. Phys. 14, p. 035010 (2012).

[3] T. Sunn Pedersen, P. Helander, E. V. Stenson, U. Hergenhahn, H. Saitoh, J. Horn-Stanja, M. R. Stoneking, J. R. Danielson, and C. M. Surko, "A new frontier in laboratory plasma- and astrophysics: electron-positron plasmas," http://www-thphys.physics.ox.ac.uk/research/plasma/JPP/papers17/pedersen_wp.pdf.

[4] H. Higaki, C. Kaga, K. Fukushima, H. Okamoto, Y. Nagata, Y. Kanai, and Y. Yamazaki, New J. Phys. 19, p. 023016 (2017).

[5] A. C. Boxer, R. Bergmann, J. L. Ellsworth, D. T. Garnier, J. Kesner, M. E. Mauel, and P. Woskov, Nat. Phys. 6, 207-212 (2010).

[6] Z. Yoshida, H. Saitoh, J. Morikawa, Y. Yano, S. Watanabe, and Y. Ogawa, Phys. Rev. Lett. 104, p. 235004 (2010).

[7] H. Saitoh, Z. Yoshida, J. Morikawa, Y. Yano, N. Kasaoka, W. Sakamoto, and T. Nogami, "Stable confinement of electron plasma and initial results on positron injection in rt-1," in NON-NEUTRAL PLASMA PHYSICS VIII: 10th International Workshop on Non-Neutral Plasmas (AIP Publishing, 2013) p. 63.

[8] C. Hugenschmidt, C. Piochacz, M. Reiner, and K. Schreckenbach, New J. Phys. 14, p. 55027 (2012).

[9] H. Saitoh, J. Stanja, E. V. Stenson, U. Hergenhahn, H. Niemann, T. Sunn Pedersen, M. R. Stoneking, C. Piochacz, and C. Hugenschmidt, New J. Phys. 17, p. 103038 (2015).

[10] E. V. Stenson, H. Saitoh, J. Stanja, H. Niemann, U. Hergenhahn, T. S. Pedersen, G. H. Marx, L. Schweikhard, J. R. Danielson, C. M. Surko, and C. Hugenschmidt, AIP Conference Proceedings 1668, p. 040004 (2015), http://aip.scitation.org/doi/pdf/10.1063/1.4923117 .

[11] J. Stanja, U. Hergenhahn, H. Niemann, N. Paschkowski, T. Sunn Pedersen, H. Saitoh, E. V. Stenson, M. R. Stoneking, C. Hugenschmidt, and C. Piochacz, Nucl. Instrum. Methods A 827, 52-62 (2016).

[12] C. Hugenschmidt, H. Ceeh, T. Gigl, F. Lippert, C. Piochacz, M. Reiner, K. Schreckenbach, S. Vohburger, J. Weber, and S. Zimnik, Journal of Physics: Conference Series 505, p. 012029 (2014).

[13] E. V. Stenson, J. Horn-Stanja, M. R. Stoneking, and T. Sunn Pedersen, J. Plasma Phys. 83 (2017), doi: $10.1017 / \mathrm{S} 0022377817000022$.

[14] D. Manura and D. Dahl, SIMION (R) 8.0 User Manual, Scientific Instrument Services, Inc., Ringoes, NJ 08551 (2008), http://simion.com/manual/.

[15] T. Sunn Pedersen, "The guiding center approximation," in Trapped Charged Particles (World Scientific (Europe), 2016) Chap. Three,, pp. 55-80.

[16] R. A. Schill, IEEE Transactions on Magnetics 39, 961-967 (2003).

[17] H. Saitoh, T. Sunn Pedersen, and the APEX Team, "A note on levitation techniques and parameter considerations towards a compact superconducting levitated dipole experiment," This Volume.

[18] E. V. Stenson, T. Sunn Pedersen, and the APEX Team, "Near-lossless charged particle injection into a magnetic dipole trap," unpublished.

[19] J. R. Danielson, D. H. E. Dubin, R. G. Greaves, and C. M. Surko, Rev. Mod. Phys. 87, 247-306 (2015).

[20] H. Saitoh, J. Horn-Stanja, S. Niß1, E. V. Stenson, U. Hergenhahn, T. Sunn Pedersen, M. Singer, M. Dickmann, C. Hugenschmidt, M. R. Stoneking, J. R. Danielson, and C. M. Surko, "Manipulation of positron orbits in a dipole magnetic field with fluctuating electric fields," This Volume. 